\title{
A Successful Approach to EduCATING Engineering LEADERS AT THE GRADUATE LEVEL
}

\author{
Professor Simon Pitts, Professor Steven Klosterman, and Professor Steven McGonagle \\ Gordon Engineering Leadership Program at Northeastern University \\ s.pitts@neu.edu, s.klosterman@neu.edu, and s.mcgonagle@neu.edu
}

\begin{abstract}
We need to develop future engineering leaders who have the capability to lead teams in efficiently and effectively delivering projects and products for their stakeholders.

This paper describes the methodology used by the Gordon Engineering Leadership Program at Northeastern University to teach Engineering Leadership at the graduate level to achieve this goal.

The approach utilized in the Gordon Engineering Leadership Program (GEL) involves an integrated course which has a close alignment with industry and leverages five primary elements: Leadership Capabilities, Leadership Laboratories, Product Development, Scientific Foundations and a Challenge Project.

This paper outlines two critical areas, the use of a leadership capability framework linked to interactive leadership laboratories and the use of mentoring to maximize the educational value of the "Challenge project".
\end{abstract}

Keywords: Engineering Leadership Capabilities, Laboratories, Product Development, Gordon Engineering Leadership

\section{INTRODUCTION}

There is a disparity between the output of our current engineering education and industry's need for young engineers who can effectively lead teams and implement engineering projects in a cross functional environment to specification, meeting performance targets, on time, and on budget to successfully achieve stakeholder needs. To bridge this gap innovative integrated approaches that develop not only technical depth but also interpersonal agility are essential to successful leadership of engineering teams [1].

The Gordon Engineering Leadership Program at Northeastern University (GEL) has been designed to close that gap. The purpose of this paper is to describe two aspects of the GEL Program: Engineering Leadership Capabilities and Laboratories, and the Mentoring of Challenge Projects.

\section{PROGRAM DESCRIPTION}

GEL is a transformational graduate program offered through Northeastern University's College of Engineering. It inculcates the psychological skills and capabilities needed to lead engineers in parallel with technical skills to successfully engineer products to customers and markets. The concentrated curriculum teaches relevant leadership theory followed by practice in leadership laboratories. Technical product development and scientific foundations courses are followed by the completion of a market-worthy challenge project. This learning framework is supplemented with three-way mentoring from industry, faculty and program mentors. The program teaches the knowledge, skills and attitudes required to successfully lead engineering teams [2].

The program curriculum is diagramed in Fig. 1, below. 


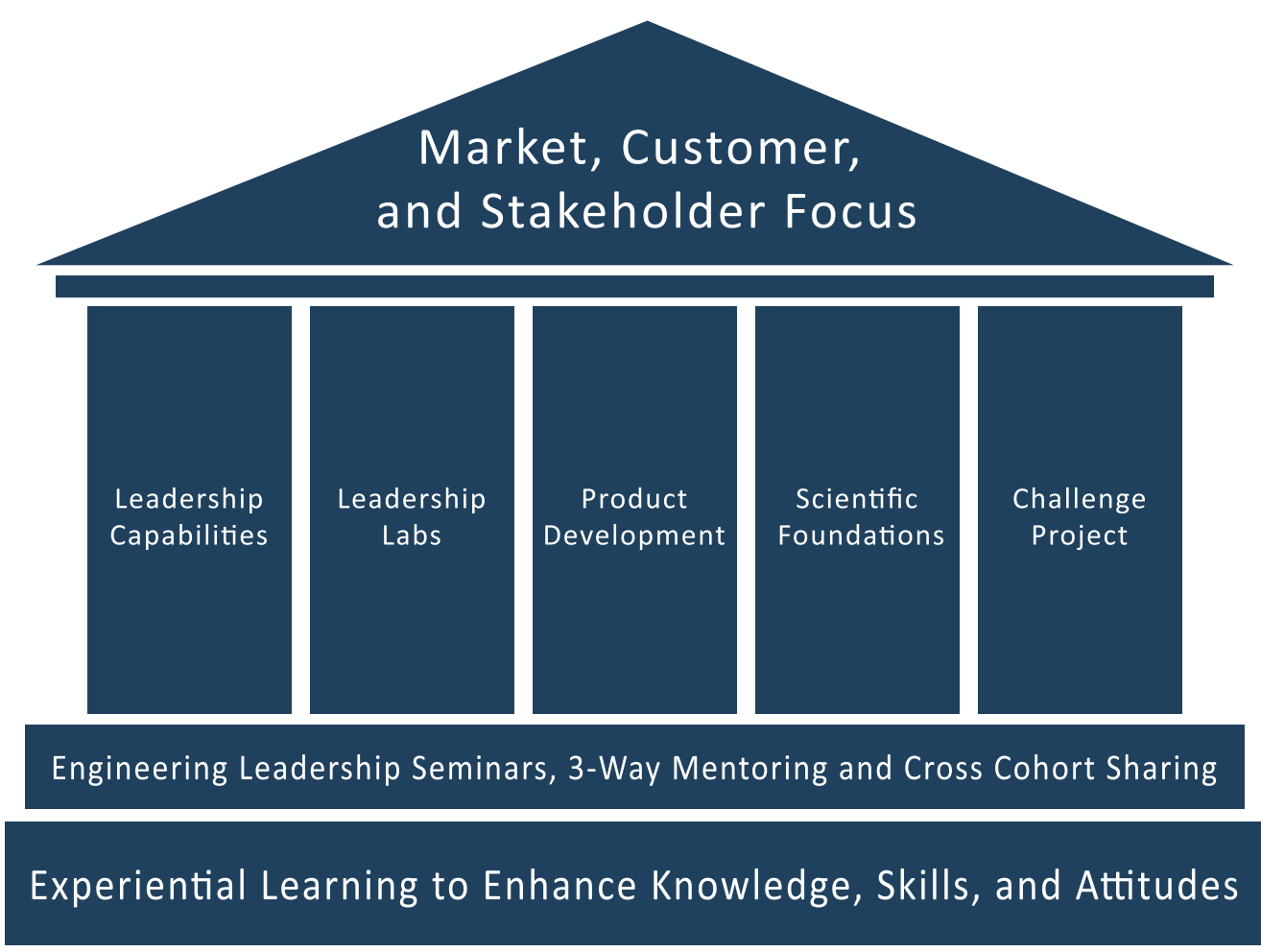

Fig. 1: The Northeastern Gordon Engineering Leadership Program

The foundation is established by four elements which are integrated into every aspect of the curriculum

- Experiential learning to enhance Knowledge, Skills and Attitudes

- Distinguished speakers from industry to discuss and model leadership

- Three-way mentoring comprised of one mentor each from the program, an industry partner and faculty from the Candidates' technical area

- Cross-cohort sharing, where Candidates learn from each other

Five pillars represent the core elements of the curriculum:

- Leadership Capabilities

- Leadership Laboratories

- Product Development

- Scientific Foundations

- Challenge Project

The foundational elements and the five key pillars all serve to develop the human and technical capabilities of engineering leadership focused on achieving the common goals of satisfying market, customer and stakeholder needs. The paper will examine two areas of the total program, the Leadership Capabilities and Leadership Laboratories, and the new way in which mentoring is used to endure the success of the Challenge Project.

\section{LEADERSHIP CAPABILITIES AND LEADERSHIP LABORATORIES}

\subsection{Key Leadership Capabilities}

The GEL leadership capabilities are derived from the Sloan-MIT Leadership model as described in the articles "In Praise of the Incomplete Leader"[3] and "Capabilities of Effective Engineering Leaders"[4], combined with proven techniques for developing leadership competencies used by the U.S. military as described in the U.S. Army Field Manual and other references [5],[6],[7].

Building upon these best practices, adapting and testing concepts in interviews and feedback sessions with industry stakeholders and advisors, GEL has identified 14 key capabilities essential to becoming an effective engineering leader:

1. Initiative: Assess risk and take the initiative, to create a vision and course of action.

2. Decision Making: Make decisions with information at hand factoring in risk; maintain and take alternative action when necessary.

3. Responsibility and Urgency to Deliver: Determination to accomplish the mission in the face of constraints or obstacles; commitment to absolute responsibility to deliver on time, pursuing necessary follow-up. 
4. Resourcefulness - Get it Done: Focus on the tasks at hand with passion, discipline, intensity and flexibility.

5. Ethical Actions and Integrity: Adherence to ethical standards and principles and the courage to act ethically and with integrity.

6. Trust and Loyalty: Commitment to actions that will instill trust and to the principle that loyalty to the team yields loyalty to the leader and vision. Working to empower those around you to make the people around you successful.

7. Courage: Face difficult/high-risk actions headon.

8. Vision: Creating compelling images of the future, identifying what could and should be for new products, systems and enterprises.

9. Realizing the Vision: Designing processes and approaches to move from abstraction to invention, innovation and implementation. Lead an organization to plan and deliver a project exercising solution judgment and critical reasoning.

10. Inquiry: Listen to others with intention of genuinely understanding their thoughts and feelings; recognize their ideas may be better than yours.

11. Interpersonal Skills: Respect needs of individuals and the group; recognition of others' strengths; coaching, gracious professionalism.

12. Communicating and Advocacy: Clearly able to explain point of view, approach to those with differing backgrounds and cultures; assess extent to which you are understood.

13. Connect - Across Disciplines, Skills and Cultures: Appreciate, engage, and connect with those who have different perspectives.

14. Negotiating and Compromise: Appreciating the need to identify potential disagreement or conflict, negotiate to find mutually acceptable solutions.

Some capabilities, such as "Responsibility" and "Urgency to Deliver", are core in developing the right attitude to drive programs to conclusion. Others, like "Inquiry" tied to empathetic listening skill development, enable the Candidates to develop the appropriate skills to better lead in diverse and stressful environments. "Vision" compels Candidates to think about the overarching goals and objectives their team is trying to achieve. "Decision Making", "Communicating and Advocacy" and "Negotiation and Compromise" cover techniques to effectively facilitate group problem solving.

\subsection{Creating Self Awareness}

Initially the Candidates participate in a series of assessment and reflection exercises designed to reveal the inner core of their own values and belief systems. Through these sessions the Candidates begin to understand the language and theory of leadership by unearthing, categorizing and describing personality traits, behavior styles, strengths and weaknesses and how they influence the way people interact with each other.

The Candidates take a Myers-Briggs Type Indicator (MBTI)[8] self-assessment to reveal their behavioral preferences in how they perceive the world and make decisions. The results are discussed and analyzed in a review session, facilitated by a certified administrator, where Candidates find their MBTI designation to be consistent with how they view themselves but they are also now sensitized to look for and recognize behavioral and personality traits in their teams.

Next, the 14 leadership capabilities are introduced to Candidates using a polar plot. This plot and descriptions of the capabilities are given to the Candidates on a card and revisited throughout the program as a method for Candidates to visualize their relative areas of strengths or weaknesses for each capability using a 10-point scale (10 being world-class, 0 being virtually inept). 


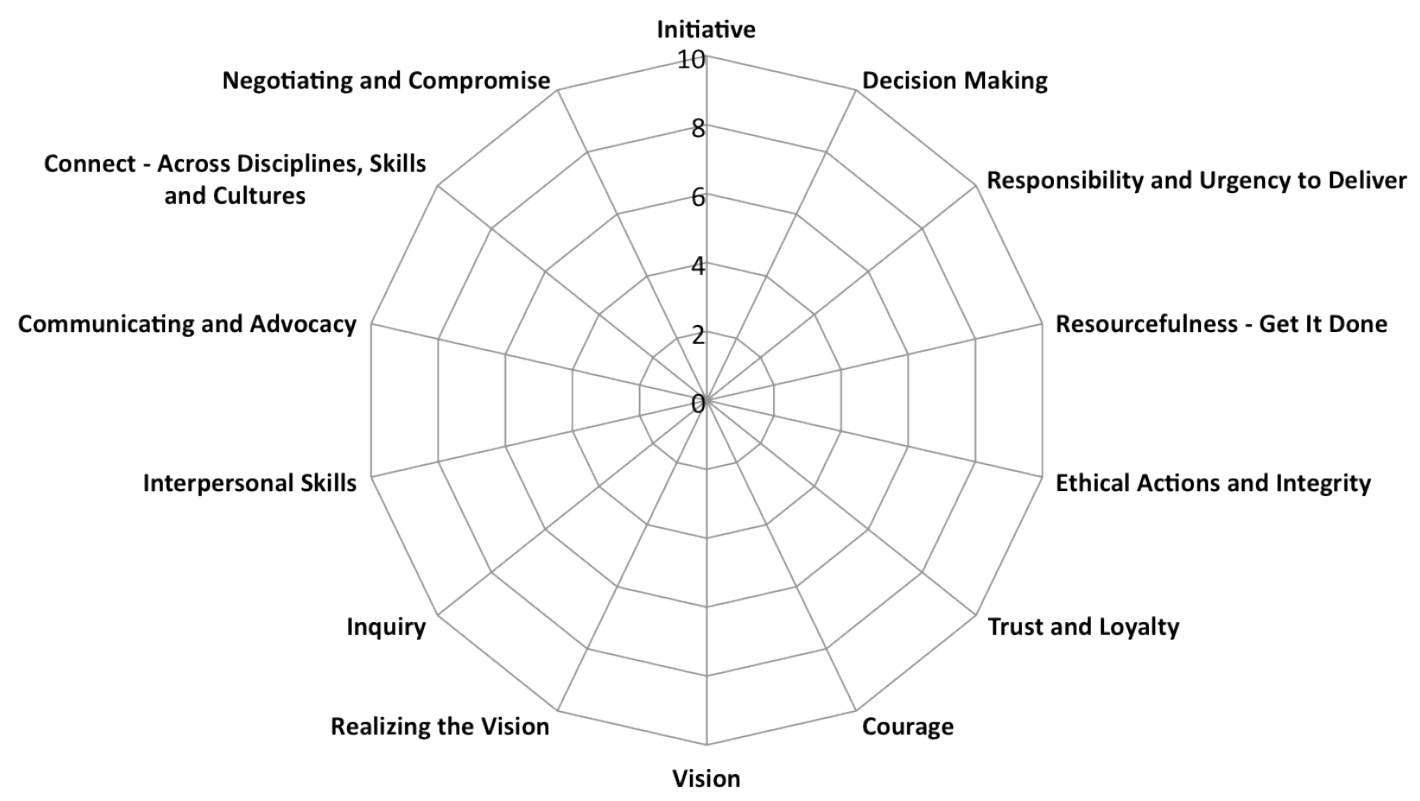

Fig. 2: Leadership Capability Polar Plot

Candidates complete a polar plot self-assessment during the first week of class. This provides a baseline for future reference and serves as a method to create individual and customized leadership development plans. The polar plot exercise is repeated later in the course augmented by additional feedback obtained from their peers, subordinates and supervisors in a comprehensive 360-degree review.

Finally, Candidates are tasked to contemplate and create a deeply self-reflective "personal mission statement" (Covey)[]. This statement of values, life's priorities and goals, considers the importance of family, work-life balance, the role of personal character in making choices and the power that comes from living one's life with purpose, integrity, and courage. This introspection serves as a "this is how I hope to live my life" manifesto and periodic review is encouraged as life changes such as promotions, job changes, family situations or crises present themselves. Candidates' feedback has indicated that is one of the more unexpected, challenging, painful and yet, ultimately rewarding and valuable exercises in the program. Most plan to continue to review and update their personal mission statement beyond graduation, integrating it into their way of assessing their lives.

Throughout the program, additional assessment tools and opportunities for reflection are introduced, including those dealing with understanding communication and conflict resolution styles. Candidates are required to submit a weekly status report in which, in addition to progress against goals, they reflect on their learning, the highs and the lows.

\subsection{The Leadership Framework}

Concurrent with the first self-assessment is an introduction to "The Leadership Framework." Candidates answer two questions, "What is a leader?" and "What does a leader do?" A facilitated discussion gradually reveals a schema covering core values, leadership knowledge, capabilities and attitudes, culminating in a group-derived definition of "leadership".

A partial example of how the framework, typically drawn on a whiteboard, is used to facilitate classroom discussion is shown in Fig. 3. 


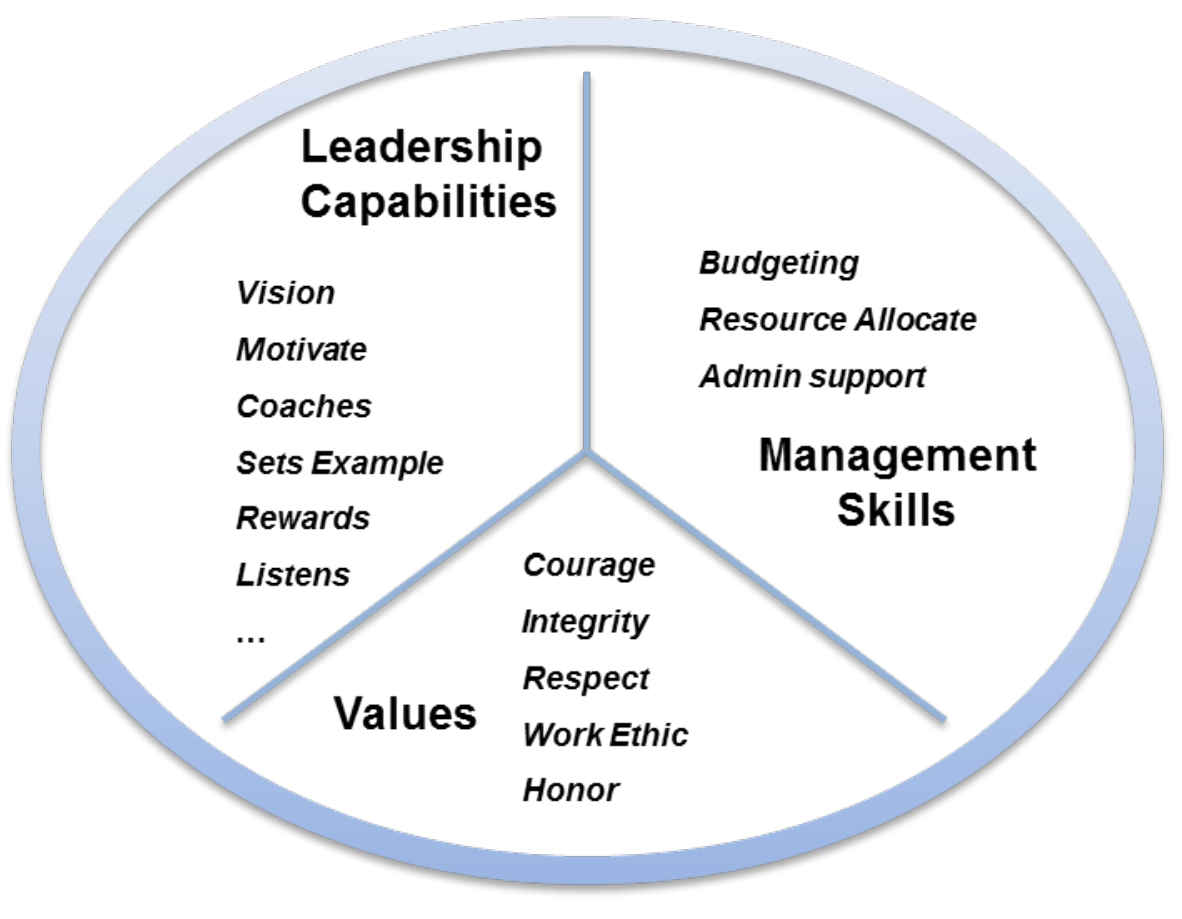

Fig. 3: Leadership Capabilities Framework

From this exercise, Candidates offer up terms that, in their own experience, seem to have some connection with leadership. Typically dozens of words randomly generated from the class are collected and categorized into one of three buckets: Values, Leadership Capabilities, or Management Skills. In the ensuing dialogue, Candidates begin to appreciate how solid values, established from one's behavior, beliefs, feelings and personality, matched with the desire and commitment to consistently embrace and live by those values, can become a source of strength.

Taken from the Leadership Framework lesson plan, the key points and learning objectives for this module include:

- Values are deeply held beliefs about right/wrong, $\mathrm{good} / \mathrm{bad}$

- Personal values are implicitly related to choice; they guide decisions by allowing for an individual's choices to be compared with each choice's associated values.

- Leadership attributes/attitudes are internal and defining qualities we possess, develop and exercise in our activities and interactions with others.

- Leadership is: influencing others by providing purpose, direction and motivation to accomplish a task while improving the organization and its people.

- Distinguishing the difference between leadership and management: Leadership deals with people and behavior; management with the science of organizing and planning programs, budgets and systems.

After having discussed, learned and practiced many leadership capabilities, there follows a contextual discussion to develop an understanding of organizational cultures and climates. This creates increased self and situational awareness, which is further codified when Candidates complete a personal SWOT (strengths, weaknesses, opportunities and threats) analysis.

\subsection{Leadership Laboratories (LLabs)}

Key to turning the concepts and theory discussed in the Leadership classroom into skills the Candidates can practice and master is the use of the highly interactive LLabs that supplement each lecture. LLabs are designed to be immersive and highly experiential to create high stress and to promote high energy by deliberately placing the Candidates outside their comfort zones but in a safe, non-threatening environment. During LLabs, Candidates depart the formal classroom setting, forming teams, groups or pairs, depending on the task and learning objectives.

For example, a class on setting standards, goals and expectations, explores the culture, climate and accepted rules of behavior of the organization and expectations as explained to a new team member, the class is followed by a LLab where Candidates are paired up to conduct an activity:

- Using scenarios, Candidates practice conducting one-on-one expectation sessions in both a send and 
receive mode. For example, when a new employee meets with their supervisor for the first time does the supervisor provide a clear description of what is expected, when and how the employee's work will be evaluated and what will be the measure of his/her success within the team?

- Candidates receive feedback from their partner and from cadre process observers and using that feedback enhance the skills by repeating the exercise with new partners.

- The result is that Candidates not only appreciate the need for these initial expectations sessions in the workplace, but they can also confidently and competently conduct such a session when required to do so.

- Linked to leadership capabilities, this exercise improves competence and confidence in interpersonal skills, communication and advocacy, inquiry, connecting across culture and disciplines, trust and loyalty and establishing and realizing a vision.

Using similar, iterative role-playing each LLab enables Candidates to practice and gain experience in performance-oriented behavior, gaining increased confidence and proficiency in using one or more leadership capabilities in a simulated real-world situation.

While most classes and LLabs focus specifically on one or more of the 14 leadership capabilities, other topics supplement them by providing targeted, peripheral lessons of significant value for an engineering leader. For example, one of these modules is entitled "Followership":

- The concept of followership is that in order to be a good leader one must first be a good follower, and that there are several key follower skills that leaders must possess. This concept is generally alien to our Candidates; in fact, the term "followership" and its interactive components is often foreign to seasoned leaders.

- Beginning with a homework reading "The Ten Rules of Good Followership," [9] Candidates report back on their recent observations and experiences, followership tenets, both good and bad are discussed and debated.

A facilitated After Action Review (AAR) closes each LLab; it is designed to reinforce the skill practiced, and demonstrate the effectiveness of learning through reflection. The AAR involves all participants and uses a series of open-ended questions to explore performance and behaviors:

- "What do you think you did well?"

- "What could you have done to improve?"

- "How did you feel when your team mate said...?"

- "What would you do differently?"

- "What did you learn?"
The AAR effectively drives home learning points by encouraging Candidates to collectively reflect and articulate what behaviors; feelings, experiences and outcomes were observed during the lab.

\subsection{Linking Leadership Capabilities to Practice}

Engineering leadership is practiced primarily in a business, corporate, research or industrial setting. Therefore, LLabs have been adapted so that case study and role-playing exercises mimic situations likely to arise in the typical work setting. This includes assigning Candidates to seek out, observe and reflect on incidents and interactions in their own work place. Example areas include:

- Conflict management

- Decision making

- Setting goals/setting expectations

- Dealing with performance issues

- Politics in the workforce

- Dealing with multi-disciplinary, multi-cultural, multi-national team issues

By studying their own workplace Candidates gain insight into how they can adapt skills to lead and influence within the unique character of their company's environment and personality.

Finally, having been broadly exposed to the skills and vocabulary associated with leadership, the focus shifts to the application of those skills in the engineering domain with assignments in which Candidates analyze specific types of team interactions within their organizations and how leadership, or lack thereof, impacts the performance of teams.

- Interacting with Marketing and other functions to collect customer needs or "voice of the customer" information to feed into the program and how those needs become specifications.

- Integrating manufacturing and service requirements as design goals and the challenge of incorporating the input from those functions at the start of a program.

- Leading teams through the dynamic of the change management, moving goals, and shifting priorities.

- Negotiating priorities and tradeoffs, team decisionmaking, coping with the tension between cost, quality and schedule pressure.

- Analyzing the company vision and how it is communicated to its members and used to inspire them.

- Understanding the role of quality and how to account for the limitations of technology, subsystems and systems to work within the envelope of tolerances and noise factors. 
Candidates are asked to seek out and interview experts and leaders within their company are able to contribute significantly to class discussions of skills application.

\section{MENTORING THE CHALLENGE PROJECT}

Significant emphasis in the program is placed on project-based learning gained through the application of the concepts and techniques learned in the classroom to the Challenge Project, which concentrates on product/process development and delivery with focus on customers and stakeholders. This project represents 50\% of the core course content and $25 \%$ of the total of the master's degree.

For the Candidate, it is the opportunity to expand his/her knowledge of a technical area and develop selfconfidence under real-world time, business, performance, and quality pressure. For the industry sponsors this is a mechanism through which a product or process of value and impact is developed on behalf of the organization.

The mentoring approach used ensures that all of these requirements are met.

A mentor is assigned to each Candidate; this mentor guides the Candidate through the entire program. As the Challenge Project is defined two additional mentors, with expertise in the project area, are selected to provide support to ensure the success of the project in satisfying the goals of the three stakeholders:

- The GEL program (Leadership and Product Development project based learning)

- The University department (Technical depth)

- The sponsoring company (impact),

This support team works together informally on an ongoing basis, but also meets regularly as a group to monitor Candidate progress. "Four-way" meetings are conducted between the Candidate and each of the three mentors to ensure alignment and communication. During the first four-way meeting, the Candidate and mentors complete a Challenge Project Mentorship Agreement, which outlines individual roles and responsibilities.

\subsection{Gordon Mentor}

The Gordon Mentor (GM) has primary responsibility for the leadership development of the Candidate. In this role, the GM coordinates the guidance and direction provided to the Candidate from other pertinent parties, including the Faculty Advisor, the Industry Sponsor/Advocate, and other individuals who influence/direct the Candidate's efforts and actions.

The GM monitors the Candidate's progress on his/her Challenge Project and provides the required guidance and direction to promote the successful completion of GEL requirements. The GM takes the lead in assisting the Candidate to meet Challenge Project timelines and reporting requirements. The GM also maintains information about Candidate performance, work quality, attitude, and any perceived challenges or problems.

\subsection{Faculty Advisor}

The Faculty Advisor (FA) assists in guiding, coaching and developing the Candidate with regard to the Challenge Project. In this role, the FA works closely with the GM to monitor progress on the Challenge Project reporting and assist with the successful completion of the Challenge Project requirements. This involves reviewing all aspects of the Challenge Project, including but not limited to: the Challenge Project Plan, Proposal, Final Report, and Presentation.

While typically the FA is a member of the faculty in the department/degree program in which a Candidate is enrolled, in special circumstances, faculty from other departments or institutions are enlisted.

\subsection{Industry Sponsor/Advocate}

The Industry Sponsor/Advocate (ISA) is an individual from the Candidate's sponsor organization who is primarily responsible for ensuring that the sponsor organization's needs are being met via the Challenge Project. In this role, the ISA is the point of contact for both the Candidate and the GM and works with both parties to arrive at mutually beneficial results from the Challenge Project deliverables.

It is critical that the ISA, serving in the advocate role, guides and mentors the Candidate while helping resolve conflicts and/or overcome obstacles the Candidate may face from internal organization sources, and ensuring harmony of effort for the achievement of the Challenge Project goals.

Experience to date shows that this system of mentoring is successful, it explicitly brings to the surface issues such as goal misalignment early enough in the project so as to enable prompt recovery actions.

\section{RESULTS TO DATE}

Candidate feedback, interviews with industry sponsors and measuring the returns of Challenge Projects clearly endorse the methods and validate the program.

\subsection{Teacher Rating and Course Evaluation (TRACE)}

The engineering leadership course and LLabs have been ranked, by Candidates, at a 4.9 out of 5 approval rating in each of the last 3 surveys. Questions relating specifically to how well lectures and in-class activities contributed to learning were ranked 4.8 and 4.9 
respectively, indicating almost universal student approval of the class.

\subsection{Student Feedback}

Candidates provided a substantial amount of written feedback on the program in course evaluations. Selected quotes include:

"GEL is very different from the various leadership programs that I have been exposed to in my career. First of all, it is not a generic leadership program that has been tailored for an application but instead is a program that was designed with the engineering leader in mind. I believe that its unique blend of technical content and real world studies in leadership is the most practical and effective method of training tomorrow's technical leaders."

"The leadership labs and activities set a strong tone for how I should act and behave as a leader. At the end of this program, not only did I learn the class material, but I have grown. I have become more confident and proactive."

"What I enjoyed most about GEL is how applicable it was to industry. I have always had a disconnect between the knowledge learned in school and what is needed in industry." "I have enjoyed how almost everything I have learned in GEL has related to a real situation I have encountered in my career."

\subsection{Industry Feedback}

Industry sponsors provide end of year assessments. Selected quotes include:

"Qualified Engineering Leadership is always in demand at <our company>. GEL greatly accelerates the learning process for new engineers and prepares them for increased responsibility at a very early stage in their engineering career. We gained an experienced engineering leader. In addition, our Candidate worked on a new product that will expand our reach in a highly competitive marketplace."

"It affords them an opportunity to run a program under the watchful eyes of their industry sponsor and the professors at GEL. These opportunities help employees learn the necessary skill sets while providing the confidence for them to take the next step in advancing their careers."

"Early development of leadership skills helps young engineers to better integrate into a variety of teams. These skills are very important in the type of fast-paced product development environment <in our company>. Students who develop leadership skills by participating in GEL are able to increase their level of contribution earlier in their career and this will ultimately help accelerate their career development."
"Dan definitely expanded both his leadership and technical skills. He expanded his leadership skills by taking ownership of larger parts of the project, which involved working with fellow team members, extended team members (including interaction with individuals in other countries), as well as working with people and organizations outside the company. Dan expanded his technical skills in many ways, including identification and development of new measurement technologies that enabled the project to succeed."

\section{SUMMARY AND CONCLUSION}

The Gordon Engineering Leadership Program at Northeastern University has developed a systemic approach to augment traditional engineering education in teaching leadership skills to improve the performance and success of projects in delivering products of genuine value to the market, company and society.

The Leadership Capabilities, Leadership Laboratories and the three-way mentoring of the Challenge Project plays a major role in the success of the Candidates' engineering leadership education.

The program continues to collect data on the impact seen by both the companies, in terms of increased productivity and value, and by the graduates, in terms of career growth and opportunities.

Initial feedback from companies and engineers participating in the program confirm that the skills developed by this teaching approach are effective and are demonstrating measurable improvement in the abilities of graduates to more efficiently and effectively lead projects to success.

\section{REFERENCES}

1 How to Lead Work Teams, Rees, 2001, Jossey-Bass/Pfeiffer 2 The Gordon Engineering Leadership Program Approach to Creating Engineering Leaders: Improving Self and Situational Awareness and Developing Targeted Leadership Capabilities Through a Leadership Framework and Leadership Labs, 2013, Pitts, Klosterman, McGonagle, 2013 ASEE Annual Conference 3 In Praise of the Incomplete Leader, Harvard Business Review, February, 2007, Ancona, Malone, Orlikowski, Senge

4 Capabilities of Effective Teams, Gordon-MIT Engineering Leadership Program, 2011,

(http://web.mit.edu/gordonelp/new.html)

5 Leadership Theory and Application sixth edition, 2004, Waesche

6 Army Leadership Fm 6-22 (Fm 22-100), 2009, US Army 7 Adaptive Leadership, 2005, Pearson Customer Publishing 8 MBTI Manual (A Guide to the Development and Use of the Myers Briggs Type Indicator), 3rd Edition, 1998, Consulting 9 The Ten Rules of Good Followership, Concepts for Air Force Leadership, 2001, Mellinger

(http://www.au.af.mil/au/awc/awcgate/au-24/meilinger.pdf) 